\title{
An Innovative Architecture of Object Detection and Tracking Using Hybrid Clustering
}

T. Mahalingam ${ }^{1}$, P. Jeyanthi ${ }^{2}$

${ }^{1}$ Sathyabama University

Jeppiaar Nagar, Rajiv Gandhi Salai

Chennai - 600 119, Tamil Nadu, India

mahaaalingam@gmail.com

${ }^{2}$ Associate Professor, Sathyabama University

Jeppiaar Nagar, Rajiv Gandhi Salai

Chennai - 600119

Tamil Nadu, India

jeyanthiprabhu@yahoo.com

ABSTRACT: In this paper we introduce a moving Object tracking models with Wireless Surveillance Camera that uses a image segmentation in color and color histogram with background subtraction for acquiring any objects in non-ideal surrounding. Here we are incorporating a competent CAMSHIFT algorithm which is combination of Normalized Cross Correlation along with MEANSHIFT algorithm. This Competent CAMSHIFT object tracking technique, which is used to imprint the object we require to concentrate, depending on the internal features of the object in the non ideal surrounding. The imprinting object is used to center any one of the tracking algorithm like Adaptive Mean Shift (CAMSHIFT), SIFT, Mean Shift, SIFT, Cross correlation algorithm and accomplish accurate real time completion of tracking moving objects is carried out through OPENCV.

Keywords: Hybrid Cluster, Normalized Cross Correlation (NCC), Competent CAMSHIFT, MEANSHIFT

Received: 10 December 2017, Revised 19 January 2018, Accepted 27 January 2018

DOI: $10.6025 /$ isej/2018/5/1/1-10

(C) 2018 DLINE. All Rights Reserved

\section{Introduction}

A significant computer visualization structure block is tracking of humans. For many applications, aligning from surveillance and military from side to side computer aided driving and improved human machine interfaces, it is needed. Human tracking's main challenges include: ${ }^{1}$ There is a dissimilarity lies in between forefront and backdrop areas; ${ }^{2}$ Distinctive objects that are tracked and equal scene of the two objects; ${ }^{3}$ Design on forefront and backdrop area the tracker is made to compare for a particular space, where for a selected region and the foremost histogram depend on itemizing the box histogram; ${ }^{4}$ Tracked objects two dimensional scaling to the scene in which the object changes their vastness from the camera; and ${ }^{5}$ Tracked objects occlusion by another object.

Information Security Education Journal Volume 5 Number 1 June 2018 